\title{
pH-Mediated Release in a Model Drug Delivery System
}

\author{
Marianne Robison, Mary R. Warmin, Clifford E. Larrabee, Jr. \\ University of Cincinnati, Cincinnati, Ohio, USA \\ ribosomn@mail.uc.edu,warminmr@mail.uc.edu, cliff.larrabee@uc.edu
}

\begin{abstract}
Targeted drug delivery systems protect healthy tissue in the patient's body while carrying the therapeutic agent to the diseased tissue. For cancer therapy, the enhanced permeability and retention (EPR) effect takes advantage of porosity of the tumor blood vessels and negligible lymphatic drainage. Nanoparticle drug carriers will accumulate in tumors without external intervention. The general problem is how best to release the drug once it has reached the targeted area. Here we show a model micellar drug delivery system that is stable and secure at a normal blood $\mathrm{pH}$ of 7.4, but disperses and releases its substrate as the $\mathrm{pH}$ drops below 7.0. The micelle is formed from the ionic surfactant, 10-undecenoate, with the hydrophobic tetrabutylammonium counterion. The pH-dependent structure and function differ markedly from other micellar solutions and suggests a key role of the counterion in both stability and solubilization power of the micelle. Our results indicate that tetrabutylammonium counterions can bring about the pH-mediated release of therapeutic agents from micellar drug delivery systems.
\end{abstract}

Keywords: micelle, solubilization, $\mathrm{CMC}, \mathrm{pH}$, model system, drug delivery, hydrophobic interactions

\section{Introduction}

Targeted drug delivery systems deliver therapeutic agents to specific parts of a patient's body. The carriers protect the drug while it circulates in the bloodstream and then release at the site of the diseased tissue. [1], [2] The enhanced permeability and retention (EPR) effect is one of the simplest approaches to targeted drug delivery for cancer treatment. Newly formed tumor vessels are leaky and lack lymphatic drainage. Nano-carriers circulating in the bloodstream will build up in the tumors without intervention. [3], [4], [5] Many tumors also have a markedly lower pH than healthy tissue due to the production of lactic acid by the anaerobic metabolism of cancer cells. This $\mathrm{pH}$ differential can trigger the release of the drug from a suitably designed carrier. [6], [7], [8]

Tetrabutylammonium 10-undecenoate (TBAU, Figure 1) micelles (Figure 2) with oil blue N (OBN, Figure 3) substrate have been shown to be an effective, simple model for isolating the molecular properties responsible for some drug delivery system functions. The hydrophobic tetrabutylammonium cation is particularly effective at stabilizing the micelle (low critical micelle concentration, CMC) and increasing its loading capacity (high solubilization power). [9] The 10-undecenoate ion is polymerizable [10] and oligomerization of the ion leads to enhanced stability of the micelle/substrate complex. [11]
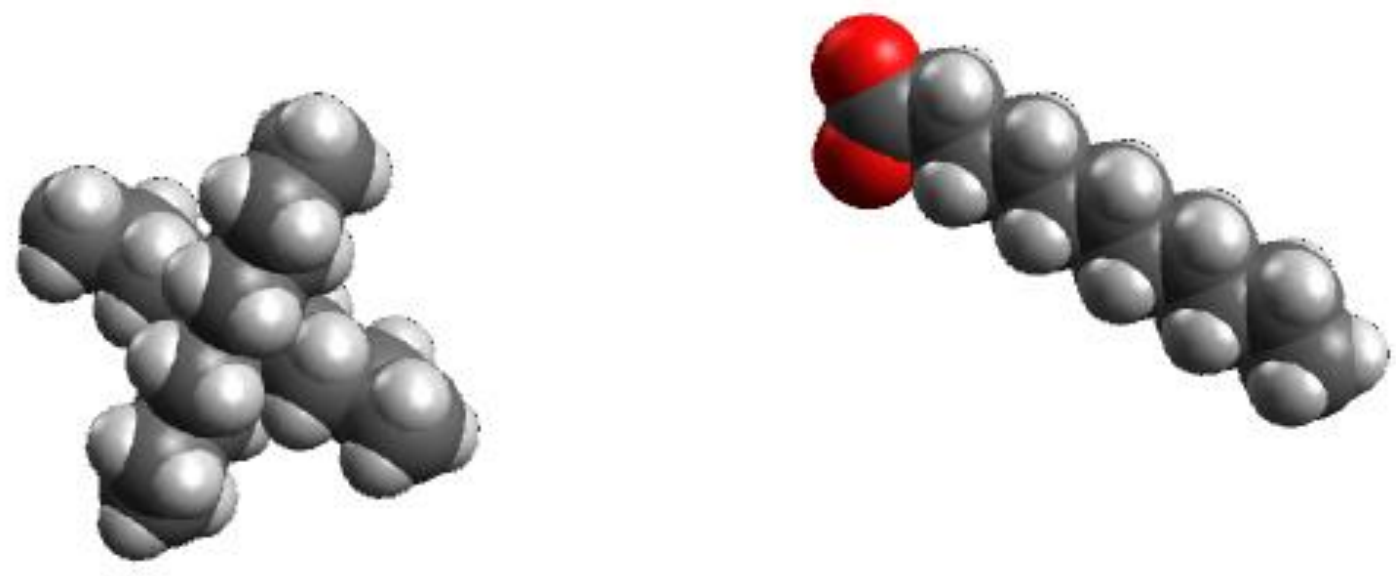

Fig. 1: Tetrabutylammonium 10-undecenoate space filling model. 


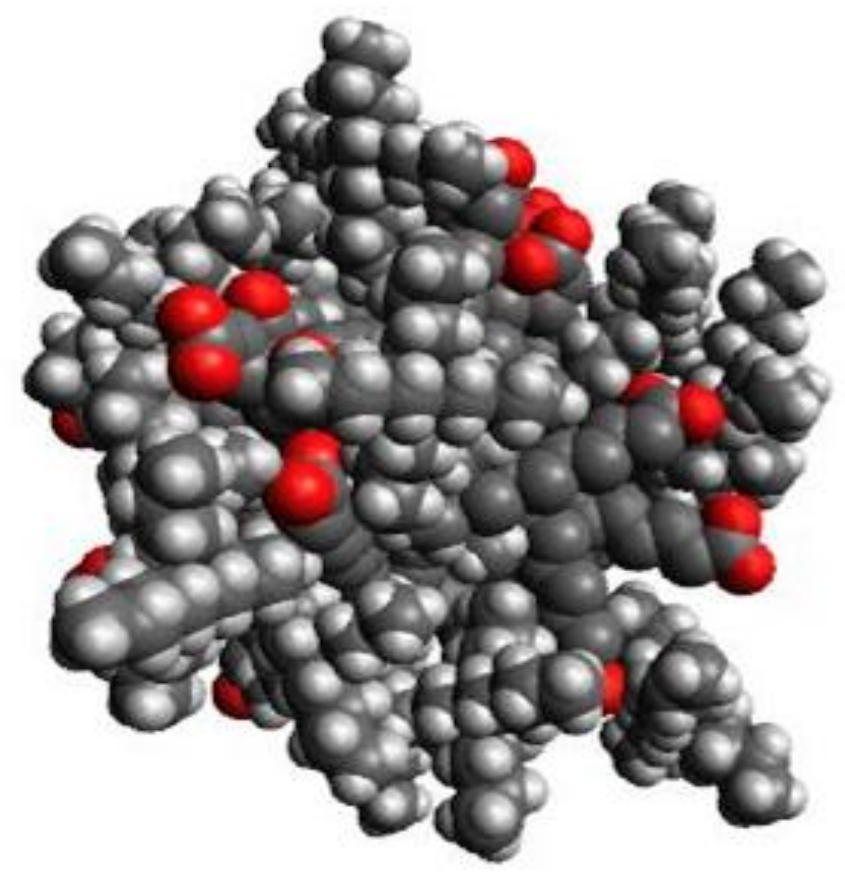

Fig. 2: Tetrabutylammonium 10-undecenoate micelle space filling model comprised of 45 10-undecenoate ions and 30 tetrabutylammonium counterions.

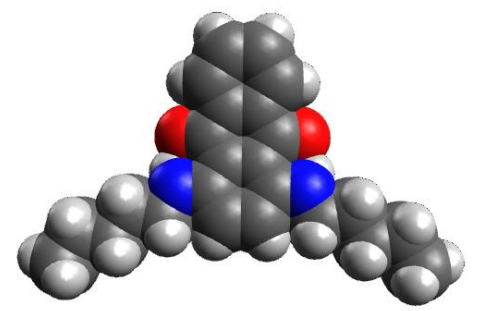

Fig. 3: Oil Blue N space filling model.

The long-term objective of this research is to determine how molecular structure influences the hydrophobic interactions that play a role in the rational design of drug delivery assemblies. The specific goal of this project was to describe the effect of $\mathrm{pH}$ on the stability and release of TBAU/OBN micelle/substrate complex.

\section{Materials and Methods}

All solutions were made up with ACS Reagent Grade, ASTM Type II water (Ricca Chemical Company). Stock solutions of tetrabutylammonium 10-undecenoate were prepared by neutralizing 10-undecenoic acid (Acros, 99\%) with tetrabutylammonium hydroxide (Acros, $40 \%$ in water) after standardizing with potassium hydrogen phthalate (Fisher, ACS). A stock solution of hydrochloric acid was prepared by dilution (Fisher, ACS, 12M) and standardized with aqueous sodium hydroxide (Ricca Chemical, ACS Reagent grade), that had been standardized with potassium hydrogen phthalate.

Absorbance measurements were made at $600 \mathrm{~nm}$ with an Ocean Optics USB4000 UV/Vis spectrometer. The $\mathrm{pH}$ measurements were made with a Vernier PH-BTA sensor calibrated with pH 4.00, 7.00, and 10.00 buffer solutions (Fisher, Certified). All measurements were made at room temperature, $295 \pm 2 \mathrm{~K}$.

\subsection{Critical Micelle Concentrations}

Solutions were prepared by adding excess oil blue N (MP Biomedicals) to the surfactant solutions, sonicating for 30 minutes, allowing to settle at least overnight, and filtering (Millipore PVDF $0.45 \mu \mathrm{m}$ ) into glass cuvettes. OBN is a water 
insoluble dye and is solubilized only in the presence of micelle solution. [9] Absorbance data for OBN in aqueous TBAU solutions were fit to the piecewise function,

$$
A= \begin{cases}0, & \text { if } c_{T B A U}<C M C \\ \left(c_{T B A U}-C M C\right) \times m, & \text { if } c_{T B A U} \geq C M C\end{cases}
$$

where $\mathrm{A}$ is the absorbance, $\mathrm{c}_{\mathrm{TBAU}}$ is the concentration of tetrabutylammonium 10 -undecenoate $\left(\mathrm{mol} \mathrm{dm}^{-3}\right), \mathrm{m}$ is the slope of the line, and $\mathrm{CMC}$ is the critical micelle concentration.

\subsection{Effect of Added Hydrochloric Acid}

Each solution was prepared by combining appropriate amounts of $\mathrm{TBAU}$ and $\mathrm{HCl}$ stock solutions, diluting to a final volume, and adding excess OBN. Solution handling and spectrometric measurements followed the same procedure as described in section 2.1. Absorbance data were fit to the function,

$$
A=\frac{a}{\left(1+\left(\frac{c_{T B A U}-c_{T B A U^{0}}}{b}\right)^{2}\right) \times\left(1+\left(\frac{p H-p H^{0}}{c}\right)^{2}\right)}
$$

where $\mathrm{a}, \mathrm{b}, \mathrm{c}, \mathrm{c}_{\mathrm{TBAU}}{ }^{0}$, and $\mathrm{pH}^{0}$, are parameters determined during the regression analysis.

\section{Results}

The experimental results and the regression lines are summarized in Figure 4. In water and up to $1 / 4$ equivalents of added $\mathrm{HCl}$, the regression lines show good linearity with well-defined $\mathrm{CMCs}$. With higher equivalents of added $\mathrm{HCl}$, the absorbance was essentially flat and equal to zero.

The CMCs are summarized in Table 1 . The first four values show a steady increase in CMC values with added $\mathrm{HCl}$. There was no evidence of micelle formation or solubilization in solutions of 0.5 equivalents of $\mathrm{HCl}$ or higher.

Table 1: CMCs for aqueous solutions of tetrabutylammonium 10-undecenoate with added hydrochloric acid.

\begin{tabular}{|c|c|c|}
\hline Equivalents of HCl added & CMC & Standard Error \\
\hline $\mathbf{0}$ & 0.029 & 0.002 \\
\hline $\mathbf{0 . 0 6 2 5}$ & 0.067 & 0.021 \\
\hline $\mathbf{0 . 1 2 5}$ & 0.099 & 0.008 \\
\hline $\mathbf{0 . 2 5}$ & 0.116 & 0.013 \\
\hline $\mathbf{0 . 5}$ & None observed & \\
\hline $\mathbf{1 . 0}$ & None observed & \\
\hline
\end{tabular}

Results of the analysis of oil blue $\mathrm{N}$ solubilization as a function of both TBAU concentration and $\mathrm{pH}$ are presented in Figure 5. Micelle solubilization is evident at high $\mathrm{pH}$, and clearly absent at low $\mathrm{pH}$.

\section{Discussion}

The CMC for TBAU without added hydrochloric acid was $0.029 \pm 0.002 \mathrm{~mol} \mathrm{dm}{ }^{-3}$. This value is in good agreement with the value reported previously [9] from solubilization, $0.031 \pm 0.003 \mathrm{~mol} \mathrm{dm}^{-3}$, and from conductivity measurements, $0.032 \pm 0.004 \mathrm{~mol} \mathrm{dm}^{-3}$.

The plots of absorbance versus surfactant concentration in Figure 1 show a distinct trend of increasing CMC with increasing equivalents of hydrochloric acid added. At 0.5 equivalents added and higher, there is no evidence of micelle formation. This indicates a destabilization of the micelle and release of the OBN with added acid. The same effect is observed in Figure 2, as $\mathrm{pH}$ decreases, the amount of OBN solubilized by micelles decreases. For any surfactant concentration, at $\mathrm{pH}$ values less than 7, most of the OBN has been released. Our results with TBAU are opposite those found for sodium dodecyl 
sulfate, where a decrease in $\mathrm{CMC}$ with decreasing $\mathrm{pH}$, was found by solubilization, conductivity, and light scattering measurements. [12]

(a)

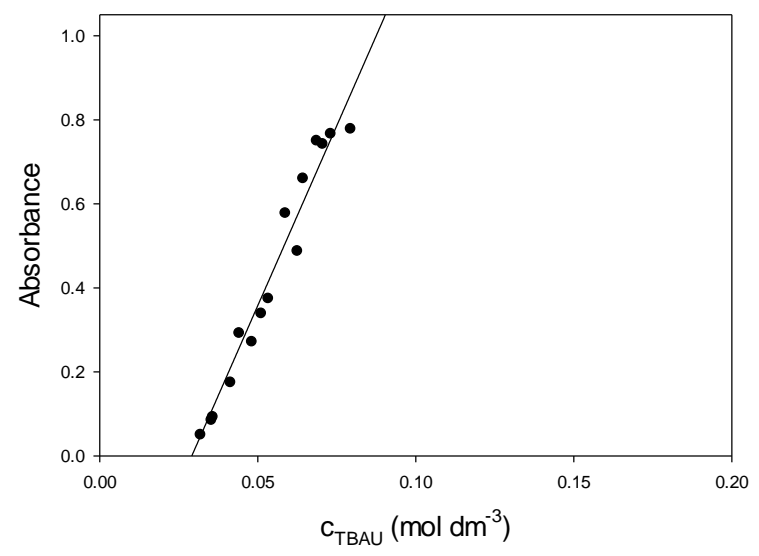

(c)

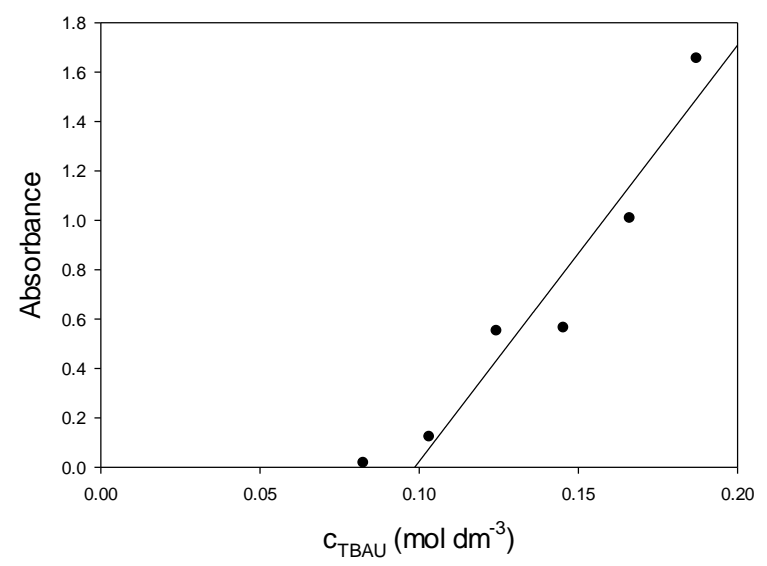

(e)

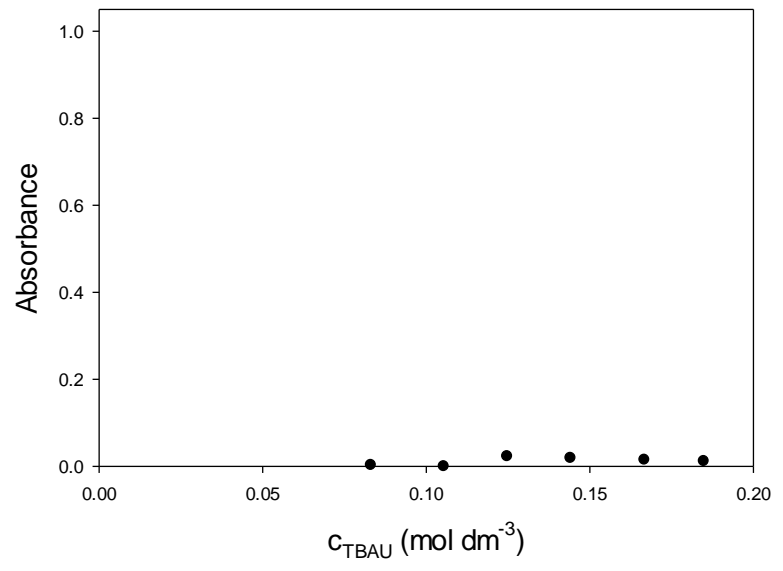

(b)

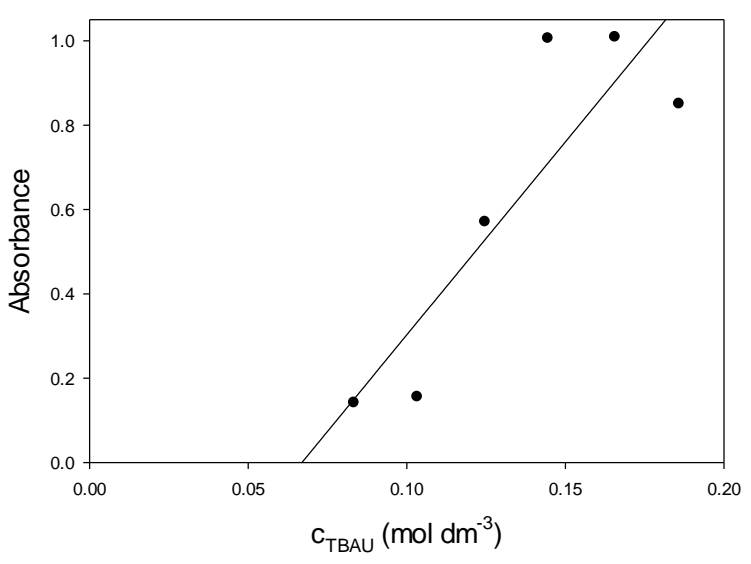

(d)

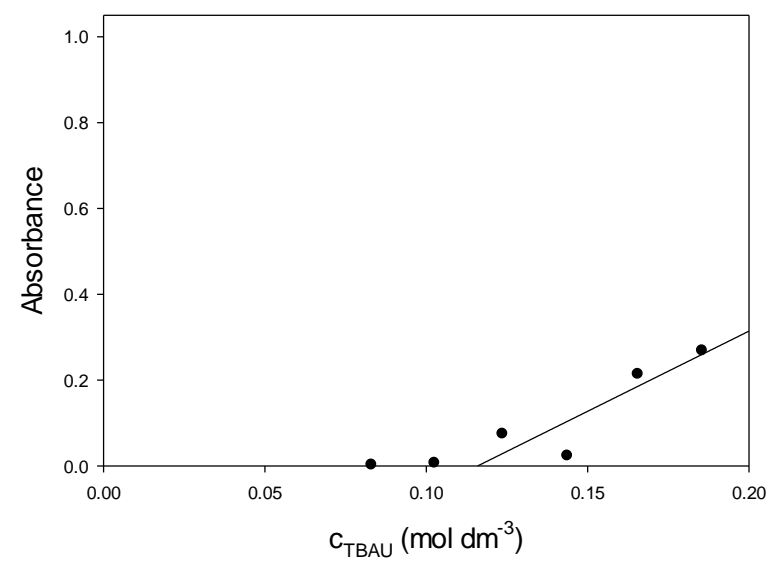

(f)

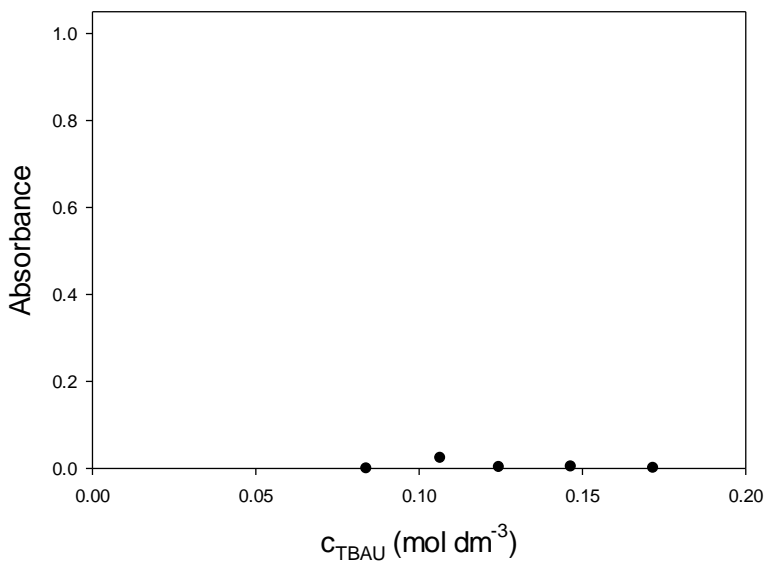

Fig. 4: Absorbance plots of oil blue $\mathrm{N}$ solubilized in aqueous solutions of tetrabutylammonium 10-undecenoates: (a) with no added $\mathrm{HCL}$; (b) with $1 / 16$ equivalents of $\mathrm{HCl}$; (c) with $1 / 8$ equivalents of $\mathrm{HCl}$; (d) with $1 / 4$ equivalents of $\mathrm{HCl}$; (e) with $1 / 2$ equivalents of $\mathrm{HCl}$; (f) with 1 equivalent of $\mathrm{HCl}$. 


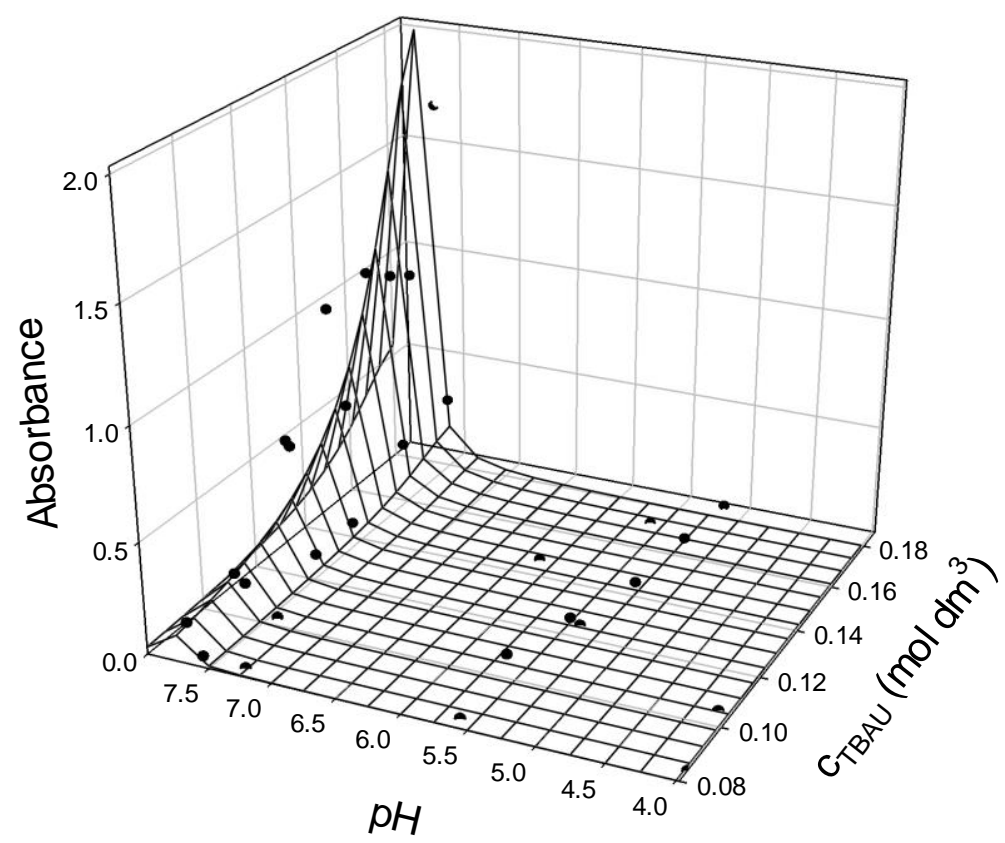

Fig. 5: Three-dimensional absorbance plot of absorbance of oil blue $\mathrm{N}$ at $600 \mathrm{~nm}$ as a function of the concentration of Tetrabutylammonium 10-Undecenoate and $\mathrm{pH}$.

Tetrabutylammonium ions have been shown to have atypical properties in aqueous solution. TBA carboxylate surfactants are highly soluble, do not exhibit clouding in the temperature range $301-371 \mathrm{~K}$ and form unusually small micelles. [13] They also exhibit an unusually high enthalpy of formation relative to homologous tetra- $n$-alkylammonium carboxylates. [14] In addition, they are the only surfactants capable of forming stable clathrate-hydrates near room temperature. [9], [15], [16]

An attempt to repeat the solubilization experiments with sodium 10-undecenoate resulted in cloudy mixtures rather than solutions suitable for the absorbance measurements. This result is consistent with earlier observations that adding acid to sodium 10-undecenoate micelle solutions yields vesicle formation [17] and the general observation that adding acid to a homogeneous surfactant solutions leads to phase separation. [18].

\section{Conclusion}

The tetrabutylammonium 10-undecenoate/oil blue $\mathrm{n}$ system has been investigated as a model for targeted drug delivery. The system is stable above $\mathrm{pH} 7$ (normal blood $\mathrm{pH}$ ), but destabilizes as the $\mathrm{pH}$ drops below 7 . This behavior contrasts with general observation that carboxylate surfactants tend to form vesicles as the $\mathrm{pH}$ is lowered [19], and with the specific case of vesicle formation by sodium 10-undecenoate [17]. The fact that TBAU/OBN destabilizes at low $\mathrm{pH}$ rather than forming an alternative aggregated phase leads to the complete release of the OBN substrate within a narrow range of $\mathrm{pH}$ values. The ability to trigger the release of the substrate with $\mathrm{pH}$ is an important feature for targeted drug delivery systems based on the EPR effect. Since many tumors are more acidic than the surrounding healthy tissue, the tumors themselves will trigger the release of the therapeutic agent. In this respect, one of the essential features of targeted drug delivery systems has been captured by the simple model system.

\section{Acknowledgements}

Marianne Robison and Mary Warmin were supported in part by the University of Cincinnati, Women in Science and Engineering (WISE) program. Mary Warmin was supported in part by the University of Cincinnati, University Research Council (URC). 


\section{References}

[1] I. Brigger, C. Dubernet, and P. Couvreur, "Nanoparticles in cancer therapy and diagnosis," Advanced drug delivery reviews, vol. 54, pp. 631-651, 2002.

[2] L. Brannon-Peppas and J. O. Blanchette, "Nanoparticle and targeted systems for cancer therapy," Advanced drug delivery reviews, vol. 64, pp. 206-212, 2012.

[3] H. Maeda, J. Wu, T. Sawa, Y. Matsumura, and K. Hori, "Tumor vascular permeability and the EPR effect in macromolecular therapeutics: a review," Journal of controlled release, vol. 65, pp. 271-284, 2000.

[4] A. K. Iyer, G. Khaled, J. Fang, and H. Maeda, "Exploiting the enhanced permeability and retention effect for tumor targeting," Drug discovery today, vol. 11, pp. 812-818, 2006.

[5] V. Torchilin, "Tumor delivery of macromolecular drugs based on the EPR effect," Advanced drug delivery reviews, vol. 63, pp. 131-135, 2011.

[6] Y. Bae, W.-D. Jang, N. Nishiyama, S. Fukushima, and K. Kataoka, "Multifunctional polymeric micelles with folatemediated cancer cell targeting and pH-triggered drug releasing properties for active intracellular drug delivery," Molecular BioSystems, vol. 1, pp. 242-250, 2005.

[7] W. Chen, P. Zhong, F. Meng, R. Cheng, C. Deng, J. Feijen, et al., "Redox and pH-responsive degradable micelles for dually activated intracellular anticancer drug release," Journal of controlled release, vol. 169, pp. 171-179, 2013.

[8] F. Huang, R. Cheng, F. Meng, C. Deng, and Z. Zhong, "Micelles Based on Acid Degradable Poly (acetal urethane): Preparation, pH-Sensitivity, and Triggered Intracellular Drug Release," Biomacromolecules, vol. 16, pp. 2228-2236, 2015.

[9] C. E. Larrabee Jr, M. R. Warmin, and M. J. Howard, "Clathrate Hydrate Formation and Micellization of Tetrabutylammonium 10-Undecenoate," International Journal of Theoretical and Applied Nanotechnology, vol. 2, pp. 441-1-6, 2015

[10] C. E. Larrabee Jr. and E. D. Sprague, "Radiation-induced polymerization of sodium 10-undecenoate in aqueous micelle solutions," Journal of Polymer Science: Polymer Letters Edition, vol. 17, pp. 749-751, 1979.

[11] C. E. Larrabee Jr., M. R. Warmin, and C. N. Iles, "Effect of Oligomerization of Sodium 10-Undecenoate on the Solubilization of a Hydrophobic Substrate," in Proceedings of the International Conference on Nanotechnology, Fundamentals and Applications, pp. 194-1-4, 2014.

[12] A. Rahman and C. Brown, "Effect of pH on the critical micelle concentration of sodium dodecyl sulphate," Journal of Applied Polymer Science, vol. 28, pp. 1331-1334, 1983.

[13] R. Zana, "Partial phase behavior and micellar properties of tetrabutylammonium salts of fatty acids: Unusual solubility in water and formation of unexpectedly small micelles," Langmuir, vol. 20, pp. 5666-5668, 2004.

[14] S. Wang and R. G. Larson, "Coarse-Grained Molecular Dynamics Simulation of Self-Assembly and Surface Adsorption of Ionic Surfactants Using an Implicit Water Model," Langmuir, vol. 31, pp. 1262-1271, 2015.

[15] Y. A. Dyadin and L. S. Aladko, "Clathrate Hydrates of Long-chain Tetrabutylammonium Carboxylates," Mendeleev Communications, vol. 5, pp. 239-240, 1995.

[16] L. Aladko and Y. A. Dyadin, "Clathrate Formation in the Tetrabutylammonium Laurate-Water System," Russian Journal of General Chemistry, vol. 69, pp. 1872-1874, 1999.

[17] J.-H. Lee, D. Danino, and S. R. Raghavan, "Polymerizable vesicles based on a single-tailed fatty acid surfactant: a simple route to robust nanocontainers," Langmuir, vol. 25, pp. 1566-1571, 2009.

[18] I. Casero, D. Sicilia, S. Rubio, and D. Pérez-Bendito, "An acid-induced phase cloud point separation approach using anionic surfactants for the extraction and preconcentration of organic compounds," Analytical Chemistry, vol. 71, pp. 4519-4526, 1999.

[19] S. Anwar and B. L. Capehart, Encyclopedia of Energy Engineering and Technology-3 Volume Set (Print). CRC Press, 2007. 